\title{
Obligaciones extraterritoriales en materia de derechos económicos, sociales y culturales. Exploraciones sobre su naturaleza y posibilidades de justificación normativa.
}

\section{Extraterritorial obligations in the field of economic, social and cultural rights. Explorations on their nature and possibilities of normative justification.}

DOI: $10.46932 / \mathrm{sfjdv2n3-015}$

Received in: May 1st, 2021

Accepted in: Jun 30th, 2021

\begin{abstract}
Dorothee Paulsen
Dirección: Westenstraße 63, 24392 Süderbrarup, Alemania

Grado académico: Magister en Derechos Humanos, Universidad Nacional de La Plata, La Plata, Argentina

Institución actual: lifeline Vormundschaftsverein im Flüchtlingsrat Schleswig-Holstein e.V., Not accompanied minor refugees rights $\mathrm{NGO/} \mathrm{ONG} \mathrm{para} \mathrm{la} \mathrm{protección} \mathrm{de} \mathrm{los} \mathrm{derechos} \mathrm{de} \mathrm{menores}$ refugiados/as no acompanados/as (Kiel, Germany) correo electrónico: dorotheepaulsen@gmail.com
\end{abstract}

\section{RESUMEN}

Surgido de la tradición política liberal, que considera al Estado como unidad soberana, el derecho internacional de derechos humanos les atribuye a los Estados las obligaciones de respetar, proteger y garantizar los derechos humanos de las personas dentro de su territorio y bajo su jurisdicción.

En el presente artículo, se defiende la tesis que este concepto de responsabilidad limitado al territorio nacional debe ser revisado, debido sobre todo a los cambios en las relaciones internacionales, por lo general subsumidos bajo el término globalización. La mayor interdependencia entre las actividades de los Estados desde la segunda mitad del siglo 20, tanto políticas, como sobre todo económicas, genera un impacto fuera del respectivo territorio nacional - impacto frecuentemente negativo para la realización de los derechos económicos, sociales y culturales (DESC) de las personas en los otros Estados (Bueno de Mesquita, Paul Hunt, Rajat Khosla, 2010). Por otro lado, también se plantea la necesidad de delimitar el alcance de la responsabilidad internacional frente a situaciones donde no se cumplen determinados DESC a nivel global, en las cuales no exista nexo causal entre las acciones de Estados externos y la vulneración de los derechos. Se plantea la necesidad de ampliar el concepto de responsabilidad en el sentido como proponen los y las defensores/as de las obligaciones extraterritoriales (ETO por sus siglas en inglés) (por ejemplo Skogly 2006, Coomans \& Künnemann 2012). La actual falta de reconocimiento y de una delimitación clara de las ETO en el derecho internacional de derechos humanos se define aquí como un vacío de protección respecto a las personas cuyos derechos se encuentran vulnerados como consecuencia de acciones y omisiones de terceros.

A partir de un análisis bibliográfico de fuentes tanto jurídicas como filosóficas, se presentará un análisis crítico del derecho vigente y de los impedimentos conceptuales allí identificados, donde se distinguen los principales impedimentos para el reconocimiento de las ETO como obligaciones legalmente vinculantes: la hegemonía del realismo en el pensamiento político y normativo, junto a las relaciones de desigualdad geopolítica. Admás exhibirán los diferentes niveles de responsabilidad desarrollados en la tesis de maestría cuyos puntos clave aquí se presentan (Paulsen, 2018), y las obligaciones correspondientes. La argumentación central a fin de justificar la necesidad del reconocimiento de las ETO procederá partiendo de la exposición de los obstáculos conceptuales dentro de la teoría de justicia de John Rawls. A continuación se presentarán a partir de seleccionados enfoques de justicia transnacional (Pogge, 
Nussbaum, O’Neill), los argumentos filosóficos en relación a los diferentes niveles de ETO nombrados líneas arriba y se esboza un cambio conceptual hacia el reconocimiento de las ETO.

Se concluye que se deben reconocer las responsabilidades más allá del territorio nacional. Para ese fin es necesario asignar las obligaciones concretas a actores lo más concretos posibles, en parte en términos de obligaciones legalmente vinculantes con instituciones de coerción. Las obligaciones concretas se deberán definir en cada caso, analizando el nivel de nexo causal, el vínculo entre las partes, y el poder relativo de ambas partes.

Palabras clave: Obligaciones extraterritoriales, Derechos Economicos, Sociales y Culturales, responsabilidad internacional, desigualdad geopolítica, derechos humanos, justicia global

\begin{abstract}
Emerging from the liberal political tradition, which considers the State as a sovereign unit, international human rights law attributes to States the obligations to respect, protect and guarantee the human rights of individuals within their territory and under their jurisdiction.

This article argues that this concept of responsibility limited to national territory needs to be revised, especially in view of the changes in international relations, usually subsumed under the term globalization. The greater interdependence between the activities of States since the second half of the 20th century, both political and, above all, economic, has had an impact outside the respective national territory - an impact that is often negative for the realization of economic, social and cultural rights (ESCR) of individuals in other States (Bueno de Mesquita, Paul Hunt, Rajat Khosla, 2010). On the other hand, there is also a need to delimit the scope of international responsibility in situations where certain economic, social and cultural rights are not fulfilled at the global level, in which there is no causal link between the actions of external States and the violation of rights. There is a need to broaden the concept of responsibility in the sense proposed by advocates of extraterritorial obligations (ETOs) (e.g. Skogly 2006, Coomans \& Künnemann 2012). The current lack of recognition and clear delimitation of ETOs in international human rights law is defined here as a protection gap with respect to persons whose rights are violated as a consequence of actions and omissions of third parties.

Based on a bibliographical analysis of both legal and philosophical sources, a critical analysis of existing law and the conceptual impediments identified therein will be presented, where the main impediments to the recognition of ETOs as legally binding obligations are distinguished: the hegemony of realism in political and normative thought, together with relations of geopolitical inequality. They will further exhibit the different levels of responsibility developed in the master thesis whose key points are presented here (Paulsen, 2018), and the corresponding obligations. The central argumentation in order to justify the need for the recognition of ETOs will proceed from the exposition of conceptual obstacles within John Rawls' theory of justice. Then, based on selected transnational justice approaches (Pogge, Nussbaum, O'Neill), philosophical arguments will be presented in relation to the different levels of ETOs mentioned above and a conceptual shift towards the recognition of ETOs will be outlined.

It is concluded that responsibilities beyond the national territory must be recognized. To this end it is necessary to assign concrete obligations to as concrete actors as possible, partly in terms of legally binding obligations with coercive institutions. Concrete obligations should be defined on a case-by-case basis, analyzing the level of causal nexus, the link between the parties, and the relative power of both parties.
\end{abstract}

Keywords: Extraterritorial obligations, economic, social and cultural rights, international responsibility, geopolitical inequality, human rights, global justice. 


\section{DEFINICIÓN DEL TÉRMINO: OBLIGACIONES EXTRATERRITORIALES}

En relación a las obligaciones de los Estados frente a violaciones de derechos humanos fuera de su territorio se refiere a obligaciones extraterritoriales. Con el término obligaciones extraterritoriales me referiré en el presente artículo a las obligaciones de los Estados respecto a los derechos humanos de las personas fuera de su territorio, (y las cuales no son ciudadanos suyos). Son obligaciones no hacia los otros Estados, sino hacia los individuos dentro de otros Estados. En el derecho internacional, las obligaciones respecto al cumplimiento de los derechos humanos son en primer lugar obligaciones hacia las personas que residen en el territorio de este Estado. Con las ETO, se introducen obligaciones más allá del territorio de un Estado, hacia las personas en territorio ajeno. Si bien las ETO tienen alcance respecto a todos los derechos humanos, aquí se indaga sobre las ETO respecto a los DESC. En lo siguiente, se refiere a las ETO tanto en situaciones donde derechos económicos, culturales o/y sociales fueron vulnerados a causa de acciones de terceros Estados (eso incluye también acciones de empresas cuyo accionar se debe regular por parte de los Estados, así se plantea), como también en situaciones donde el vínculo causal no existe. Este alcance coincide con el alcance de las ETO en los Principios de Maastricht sobre las obligaciones de los Estados en el Área de DESC.

\section{DESCRIPCIÓN DEL PROBLEMA: SITUACIONES DE VULNERACIÓN DE DERECHOS SIN POSIBILIDAD DE RECLAMO/REMEDIO}

Se pueden mencionar diferentes situaciones a fin de ejemplificar el vacío de protección aquí planteado. Existen por un lado situaciones donde los Estados domésticos no son capaces de garantizar los DESC dentro de su territorio. Se supone que deben emplear al máximo sus recursos disponibles para garantizar progresivamente estos derechos. Además se supone que deben pedir ayuda a la comunidad internacional (art. 2(I) PIDESC). Eso tanto en situaciones de pobreza persistente como en situaciones de emergencias humanitarias a causa de catástrofes naturales o bien de guerras. El debate en mencionadas situaciones prevalece acerca de si existen obligaciones extraterritoriales de la comunidad internacional, por parte de los Estados tanto colectivamente como de cada uno, de colaborar con la realización de los derechos de las personas en otro Estado. No existe unanimidad ni delimitación de las obligaciones respecto a tales situaciones (Coomans, Langford 2013; de Schutter 2012). De esta manera se suele considerar sobre todo por parte de los Estados en condiciones de asistir, que existen obligaciones generales de todos los Estados, pero a modo voluntario: obligaciones de caridad, no reclamables por las personas cuyos derechos se vulneran.

Otras situaciones se presentan donde existen acciones u omisiones posibles de ser reconstruidas y adjudicadas a determinados Estados. Por ejemplo la venta subsidiada de partes de pollo a países africanos, 
donde los campesinos locales no pueden competir con los precios y pierden su sostén de vida, se vulneran los derechos al trabajo y a la alimentación. Además, en esta situación se pone en riesgo el derecho a la salud, ya que no existen cadenas de frio para asegurar la calidad del alimento (Coomans \& Künnemann 2012). Otro ejemplo es el caso de una mina de oro de la empresa Goldcorp, con sede principal en Canadá, que se instaló en territorio indígena en Guatemala. A consecuencia, la población indígena sufrió daños respecto al estado integro de su territorio y sus posibilidades de trabajo, como cultivo. Además se contaminó el agua del rio dentro del territorio, el cual consume la población (derechos a la alimentación, al trabajo, al agua, a la salud) (Coomans \& Künnemann 2012).

En relación a estas diferentes situaciones, se elaboraron categorías de responsabilidad:

La responsabilidad indirecta: Situaciones sin nexo causal: donde la vulneración de los derechos no se generó a causa de acciones de terceros. Las obligaciones extraterritoriales se denominan obligaciones indirectas colectivas (de todos los Estados). El ejemplo mencionado es la situación de catástrofe natural.

La responsabilidad directa difusa: Se considera que la situación de pobreza persistente es diferente a las situaciones de responsabilidad indirecta mencionadas líneas arriba: se propone una redefinición de estas situaciones, frecuentemente consideradas como no causadas por acciones de terceros. Aquí se propone definir estas situaciones como tales donde el sistema de comercio internacional, funcionando a través de reglas injustas para determinadas regiones y determinados Estados, causa por lo menos en gran parte la pobreza persistente. La responsabilidad aquí se denomina difusa porque no se puede adjudicar responsabilidad a nadie en concreto sino a varios actores de manera difusa. Le corresponden obligaciones extraterritoriales colectivas, sobre todo de los Estados más poderosos, de reformar el sistema de comercio internacional.

La responsabilidad directa: esta a su vez esta subdividida en responsabilidad directa individual y responsabilidad directa colectiva. Se refiere a situaciones como las mencionadas líneas arriba: El caso de la mina de oro es un caso de responsabilidad directa individual (Canadá), el caso de la venta subsidiada de partes de pollo un caso de responsabilidad directa colectiva (Estados de la Unión Europea).

En todas las situaciones mencionadas se plantea por parte de los que abogan por el reconocimiento de las ETO que se debería reconocer responsabilidad de los Estados externos, sin perjuicio de la primordial responsabilidad del Estado doméstico.

Sin embargo, un análisis del derecho vigente no da respuesta positiva a este reclamo. Tradicionalmente, en tales situaciones se le adjudica únicamente al Estado doméstico responsabilidad de respetar, proteger y garantizar los derechos. Los que critican esta postura tradicional mencionan la 
imposibilidad o enorme limitación de determinados Estados, por su situación desigual a nivel geopolítico, por ejemplo de negociar los términos de los contratos de importación y exportación de alimentos o las condiciones de trabajo en empresas multinacionales dentro de su territorio.

No hay unanimidad sobre la naturaleza de las obligaciones extraterritoriales, si son obligaciones legalmente vinculantes o solamente obligaciones de caridad. Las disposiciones en los instrumentos de derechos humanos, sobre todo en el PIDESC, son muy generales. El artículo más expreso respecto al tema, el art. 2 (I) PIDESC, no aclara si existe una obligación legalmente vinculante de brindar cooperación y asistencia internacional. Aún en el caso de considerar que tales obligaciones existen, no delimita a quién se podría reclamar su cumplimiento o la reparación de daños.

Se identifican como obstáculos conceptuales dentro del derecho la soberanía y la jurisdicción, surgidos de la tradición liberal del Estado. Estos conceptos son principios fundamentales del derecho internacional desde la Paz de Westfalia. Por lo general, se consideran casi como sinónimos, interpretando la jurisdicción de un Estado como coincidiendo con el territorio soberano. La responsabilidad de los Estados se considera limitado al territorio soberano, fuera del mismo se sitúa el límite de la responsabilidad (Skogly 2006).

Más allá de los avances y la evolución constante del derecho, mencionando al respecto la formulación de los Principios de Maastricht sobre las obligaciones extraterritoriales de los Estados en el Área de los DESC (2012) y la mención cada vez más frecuente de las ETO en las Observaciones Generales del Comité de DESC, faltan una delimitación clara del alcance de las obligaciones extraterritoriales, como también instrumentos e instituciones de monitoreo y sanción.

Al respecto de esta falta de claridad y delimitación, donde prevalece el debate por la naturaleza y el contenido concreto de las normas, y donde se aboga por la introducción de nuevas normas dentro del derecho, la filosofía política y del derecho puede aportar tanto un análisis y una revisión crítica del derecho vigente, como argumentos para sostener la necesidad de las ETO.

\section{PRESENTACIÓN DE IMPEDIMENTOS CONCEPTUALES DENTRO DE LA FILOSOFÍA POLÍTICA PARA EL RECONOCIMIENTO DE LAS ETO Y DE ARGUMENTOS A FAVOR DE SU RECONOCIMIENTO.}

El sistema internacional de protección de los derechos humanos tal como se ha constituido al momento de su creación, y como está constituido en la actualidad, tiene sus raíces en la tradición de la teoría del contrato. En esta teoría, se concibe al Estado como condición necesaria para la posibilidad de derechos. El contractualismo justifica al Estado en un contrato entre las personas, quienes acuerdan libremente en salir de un supuesto estado de naturaleza, y formar una sociedad bajo el mando de un 
soberano, o reglas, o principios que acordaron libremente, y que restringen su libertad por decisión propia. Las condiciones de justicia se describen de diferentes maneras, sin embargo, generalmente se supone que, para que la justicia sea posible y necesaria, deben existir las siguientes condiciones: las personas coexisten al mismo tiempo y en un territorio geográfico bien definido. Las personas en el estado de naturaleza son (más o menos) iguales en fuerza y poder, por lo cual nadie puede dominar a los demás; y las personas son independientes y auto interesadas, aunque no sean necesariamente egoístas. Dentro de esta tradición, la posibilidad de justicia se limita al Estado nacional. Más allá del Estado no existen las condiciones de justicia, por lo cual no puede haber justicia. Fue I. Kant quien formuló los pasos a seguir para mantener la paz entre las naciones, a través de una federación de los pueblos (Kant, 1795). De allí nace la idea para la creación de lo que hoy son las Naciones Unidas. Sin embargo, en esta federación entre Estados, si bien se reconocen los derechos universalmente, se adjudica la responsabilidad por su garantía, protección y respeto a los Estados nacionales. No se amplían las condiciones de aplicación de la justicia al nivel internacional. El filósofo John Rawls se enmarca dentro de esta tradición contractualista, modificando significativamente el rol del contrato y elaborando una teoría de justicia que revolucionó la teoría de justicia dentro de la sociedad. Aquí se debe mencionar sobre todo el principio de diferencia que la teoría de justicia de Rawls introduce (Rawls 1971), y según el cual las desigualdades dentro de una sociedad solo se pueden justificar sí resultan de un orden que beneficia a los menos privilegiados dentro de la sociedad. En este sentido introduce la necesidad de justificar desigualdades en las posiciones dentro de una sociedad. Sin embargo, al transportar su teoría al nivel internacional, este principio no tiene aplicación. Lo que critican las y los filósofos quienes abogan por la necesidad de aplicar la justicia socioeconómica a nivel internacional, es justamente esa diferencia entre el nivel doméstico y el nivel internacional. Sostienen que a nivel internacional están dadas las condiciones de justicia socioeconómica. J. Rawls considera que a nivel internacional no existen instituciones a las que se aplican los principios de justicia (Rawls 1999): no hay lugar para organizaciones multinacionales, para instituciones internacionales. En ese sentido, su teoría a nivel global se puede ubicar dentro del paradigma realista, uno de los principales paradigmas en las relaciones internacionales, donde se supone que el Estado es independiente de los demás Estados, y persigue solamente su interés nacional al actuar a nivel internacional. Las instituciones internacionales no tienen relevancia argumentativa para el realista. Entre los Estados nacionales domina la anarquía. No existe lugar para normas éticas de acción: De manera similar supone Rawls en el contrato entre los representantes de los Estados que los Estados son independientes, iguales en poder, auto interesados y autosuficientes. A partir de esas condiciones determinan los principios de justicia para el nivel internacional. De acuerdo con Beitz, Pogge, Nussbaum, 
O`Neill se considera aquí que este modelo no se ajusta a la realidad: Los Estados no son independientes, iguales en poder y autosuficientes.

Se sostiene que es necesario elaborar un marco conceptual distinto para el nivel internacional. Frente a las ideas expresadas dentro del paradigma realista o del contractualismo liberal, que son la base de las decisiones políticas tomadas dentro de los Estados más poderosos y dentro de las organizaciones intergubernamentales como las Naciones Unidas, el Banco Mundial y el Fondo Monetario Internacional, se presentan aquí propuestas distintas: Estas resaltan las relaciones asimétricas de poder, la interdependencia y la incapacidad de los miembros de los Estados más marginados, de ejercer sus capacidades y poder rechazar propuestas que no les resultan convenientes. Y se plantea que la aplicación de la justicia socioeconómica solamente a nivel doméstico no se puede justificar con argumentos éticos, sino que se justifica con razones de nacionalismo, de preferencia moral a nivel nacional, lo cual se plantea dentro del paradigma realista.

Los enfoques de justicia global a los que se refiere aquí apuntan a un cambio de la manera de concebir la responsabilidad y las obligaciones que se desprenden de ella a nivel global. Presentan fundamentos a favor de una ampliación de las obligaciones más allá de las fronteras nacionales, concibiendo a la esfera internacional como ámbito donde la justicia puede y debe ser aplicada. Los fundamentos para tal ampliación difieren: Pogge (2006) sostiene que existen a nivel global instituciones de coerción que impactan significativamente en la vida de todos, y sobre todo tienen un impacto perjudicial en las vidas de las personas más pobres. Mejor dicho, su pobreza es consecuencia de la manera en la cual las instituciones internacionales reparten los bienes. Su argumento es que este impacto negativo debe ser corregido, tanto a través de reformas profundas de las instituciones, como de la introducción de un impuesto que sirva para realizar una distribución justa de la riqueza. Nussbaum (2007) identifica como erróneo el concepto de la justicia como recíproca dentro de un marco institucional creado para iguales en poder y fuerza, independientes y autosuficientes. Sostiene que el requerimiento de la justicia es realizar las capacidades identificadas como básicas para todas las personas individualmente. E identifica la estructura institucional actual a nivel global como no apta para cumplir con este requerimiento. Plantea que las relaciones internacionales deben ser organizadas y reguladas para realizar las capacidades a nivel global, ya que existen dependencias a nivel global, y hay muchos actores que impactan en las posibilidades de realizar las capacidades básicas de las personas, a nivel internacional y nacional. O’Neill (2007) sostiene que la justicia debe regir en condiciones donde una pluralidad de agentes interactúa, y como constata que hay tal interacción a nivel global, a estas relaciones se deben aplicar principios de justicia. Los enfoques respaldan un cambio de la concepción de las obligaciones extraterritoriales desde una concepción en términos de la ayuda humanitaria por razones de caridad, considerada como arrogancia y 
que genera mayor dependencia, hacia la concepción de obligaciones y derechos, por razones de justicia. Así concibe Pogge las obligaciones a nivel global como obligaciones negativas, de justicia correctiva; y las concibe en términos de obligaciones a las cuales corresponden derechos. $\mathrm{O}^{\prime}$ Neill desarrolla un concepto de obligaciones negativas, pero cuya realización requiere de una estrategia dual: por un lado, formula los principios de acción negativos de no engaño y no coerción que aplican a nivel universal. Por otro lado, plantea que la exposición al engaño y la coerción dependen del poder relativo, el cual por tanto debe ser equilibrado a través de diferentes acciones de prevención. El enfoque de Nussbaum difiere al ser un enfoque teleológico, en el cual la justicia requiere la realización de las capacidades humanas. Identifica principios políticos a nivel global que sostienen que la responsabilidad por la realización de las capacidades debe estar adjudicada a todos, sobre todo a los más poderosos, no solamente Estados, sino también empresas multinacionales y organizaciones internacionales. Abre así también un camino para introducir responsabilidades de los nuevos actores con gran poder a nivel global, como son las empresas transnacionales.

A partir de las propuestas de justicia transnacional seleccionadas, se pueden sustentar obligaciones extraterritoriales en el área de los derechos económicos, sociales y culturales.

Las instituciones cuya creación se exige respecto a las obligaciones extraterritoriales en todas las situaciones, no colisionan con el principio de soberanía, por diferentes razones. Para O`Neill, justamente, la justicia requiere empoderar a los que no pueden realmente ejercer su soberanía, porque no tienen posibilidades de rechazar propuestas engañosas. En la situación de deprivación y de gran desventaja económica y política respecto al poder de negociar, las obligaciones de respetar y proteger los deberes de no engaño y no coerción justamente se convierten en más fuertes para los más poderosos, exigir que se tenga en cuenta la situación real, no abstracta, del otro Estado, no es faltar el respeto a su soberanía, sino darle la posibilidad de ejercerla. Para Pogge y Nussbaum, la soberanía puede ser distribuida de manera vertical.

Por todo lo antedicho, se concluye que se deben reconocer las responsabilidades más allá del territorio nacional, y que la tarea pendiente para este reconocimiento es la asignación de las obligaciones concretas a actores lo más concretos posibles, en parte en términos de obligaciones legalmente vinculantes con instituciones de coerción. Las obligaciones concretas se deberán definir en cada caso, analizando el nivel de nexo causal, el vínculo entre las partes, y el poder relativo de ambas partes, para saber cuál es exactamente la responsabilidad de cada uno. 


\section{BIBLIOGRAFÍA}

Beitz, C. (1979). Political Theory and International Relations. Princeton: Princeton University Press.

Bueno de Mesquita, J. e. (2010). The Human Rights Responsibility of International Assistance and Cooperation in Health. In M. Gibney, \& S. Skogly, Universal Human Rights and Extraterritorial Obligations (pp. 104-129). Philadelphia, Pennsylvania : University of Pennsylvania Press.

Coomans, F., \& Künnemann, R. (2012). Cases and Concepts on Extraterritorial Obligations in the Area of Economic, Social and Cultural Rights. Cambridge: Intersentia.

De Schutter, O. e. (2012). Commentary to the Maastricht Principles on Extraterritorial Obligations of States in the area of Economic, Social and Cultural Rights. Advanced uneditd version.

Kant, I. (1795/2002). Zum ewigen Frieden. Stuttgart: Reclam .

Langford, M., \& al., e. (2013). Global Justice, State Duties. The extraterritorial scope of economic, social and cultural rights in international law. Nueva York: Cambridge University Press.

Nussbaum, M. C. (2007). Frontiers of Justice. Disability, Nationality, Species Membership. Cambridge Massachussets: Harvard University Press, Cambridge Massachussets .

O`Neill, O. (2007). Bounds of justice. Cambridge: Cambridge University Press.

O’Neill, O. (2002). Hunger, Needs and Rights. In C. Boyd, Extra-National Obligations. A Survey of Moral Philosophy. Working Paper. International Council on Human Rigths Policy.

O`Neill, O. (1996). Justicia, Sexo y Fronteras Internacionales. In M. C. Nussbaum, La Calidad de Vida (pp. 393-419). México D.F.: Fondo de Cultura Económica.

Paulsen, D. (2018). Obligaciones extraterritoriales en materia de derechos económicos, sociales y culturales: aportes desde la filosofía. http://sedici.unlp.edu.ar/handle/10915/71690

Pogge, T. (2013). Hacer Justicia a la Humanidad. México, D.F.: Fondo de Cultura Económica.

Pogge, T. (2006). World Poverty and Human Rights. Maldon, USA: Polity press.

Rawls, J. (1975). Eine Theorie der Gerechtigkeit. Frankfurt am Main: Suhrkamp.

Rawls, J. (1999). The Law of Peoples. Cambridge Mass.: Harvard University Press. 
Skogly, S. (2006). Beyond National Borders: States' Human Rights Obligations in International Cooperation. Antwerpen/Oxford: Intersentia. 